Bulletin of Pharmaceutical Sciences
Assiut University
Website: http://bpsa.journals.ekb.eg/
e-mail: bullpharm@aun.edu.eg

\title{
REAL-WORLD SAFETY AND EFFICACY OF TICAGRELOR IN PATIENTS WITH ST SEGMENT ELEVATION MYOCARDIAL INFARCTION AT ASSIUT UNIVERSITY HEART HOSPITAL
}

\author{
Huda AlSayed Mohammed ${ }^{1}$, Mohamed Aboel-Kassem F. Abdelmegid ${ }^{2}$, Ahmed Mostafa \\ Alsayed $^{3}$ and Mostafa A. Sayed $\mathrm{Ali}^{4^{*}}$ \\ ${ }^{1}$ Clinical Pharmacist, Coronary Care Unit, Assiut University Heart Hospital, Assiut, Egypt \\ ${ }^{2}$ Department of Cardiovascular Medicine, Faculty of Medicine, Assiut University, Assiut, \\ Egypt \\ ${ }^{3}$ Department of Pharmaceutics, Faculty of Pharmacy, Assiut University, Assiut, Egypt \\ ${ }^{4}$ Department of Clinical Pharmacy, Faculty of Pharmacy, Assiut University, Assiut, Egypt
}

\begin{abstract}
Objectives: The new generation antiplatelets are generally recommended by the current US and European guidelines. This article aimed to address the efficacy and safety of using ticagrelor in patients with ST-elevated acute coronary syndromes.

Methods: This was a prospective observational study on 120 patients admitted to the Cardiology Care Unit of Assiut University Hospital with ST-segment elevation acute coronary syndrome, who were admitted for urgent revascularization and received aspirin and the P2Y12 antagonist ticagrelor. The patients were followed for three months between May 2018 and September 2019. The primary efficacy end point was the incidence of major adverse cardiovascular events (MACE).

Results: At three months, the primary efficacy end point, a composite of cardiovascular death, myocardial infarction, or stroke - occurred in 11/120 (9.2\%) patients. The primary safety end point, the incidence of major bleeding, was observed in two patients (1.7\%) with no fatal bleedings. Minor and minimal bleeding occurred in $2.5 \%$ and $20.8 \%$ of patients, respectively. Other efficacy endpoint was myocardial infarction $4.2 \%$ and stent thrombosis (1.7\%). Severe dyspnea found in $5.0 \%$ patients and led to $1.7 \%$ ticagrelor discontinuation. Multivariate regression analysis revealed no association between bleeding and patients' age, sex nor IV administration of glycoprotein IIb/IIIa inhibitor.

Conclusion: The MACE with ticagrelor was higher than previously reported in most studies. Dyspnea rate was higher and more pronounced than bleeding rate. The majority of bleeding cases was minimal with no fatal major bleeding and no bleeding led to comorbidity.
\end{abstract}

\section{INTRODUCTION}

Ischemic heart disease (IHD) is considered the single leading cause of death worldwide which accounted for a combined 15.2 million deaths in $2016^{1}$. Egypt is ranked third in IHDrelated deaths among lower-middle-income countries according to the data of the World Health Organization $2010^{2}$. Dual antiplatelet therapy (DAPT) has a mandatory role in the management of IHD patients for its proven benefit of prevention of further ischemic cardiac events and reduction in mortality rates ${ }^{3}$.

Aspirin and clopidogrel DAPT have being used as the mainstay DAPT in Egypt; yet many fundamental genetic and clinical problems exist ${ }^{4}$. Clopidogrel has a relatively low bioavailability in which less than $10 \%-15 \%$ of the administered dose becomes an active $\mathrm{P} 2 \mathrm{Y} 12$ inhibitor and plasma concentration is further decreased in low creatinine clearance ${ }^{5 \& 6}$. In addition to the slow onset of 
platelet inhibition and different genotyping with high rate of CYP2C19 loss-of-function (LOF) alleles in Egyptian individuals ${ }^{7}$. This genotyping manifested as delayed onset/offset of action and incomplete inhibition of platelet aggregation $^{7 \& 8}$. The new generation of $\mathrm{P}_{2} \mathrm{Y}_{12}$ inhibitor antiplatelet ticagrelor and prasugrel are generally recommended by the current American College of Cardiology/American Heart Association (ACC/AHA) and European Society of Cardiology/European Association for Cardio-Thoracic Surgery (ESC/EACTS) over clopidogrel for ACS management ${ }^{3 \& 9}$.

Ticagrelor is a reversible and direct-acting antiplatelet that provides rapid inset/offset onset of action with more pronounced and consistent P2Y12 inhibition ${ }^{10}$. In a broad population of patients with acute ST-elevated myocardial infarction (STEMI) managed by primary percutaneous coronary intervention (PPCI), ticagrelor reduced death risk resulting from vascular causes, myocardial infarction (MI), or stroke during first year of index event without increasing the overall risk of major bleeding $^{11}$. There were also reductions in cardiovascular and total mortality, MI, and stent thrombosis ${ }^{12}$.

The recent introduction of ticagrelor in the Egyptian market along with the current global guidelines drove physicians to substitute clopidogrel with ticagrelor for many patients. However, real-world evidence of efficacy and safety and therapeutic response of ticagrelor in Egyptian patients with STEMI who are managed by PPCI is limited.

This study aimed to explore and monitor the safety and efficacy of ticagrelor in Egyptian patients with STEMI and treated with PPCI at Assiut University Heart Hospital.

\section{Ethical approval}

This study was approved by the Research Ethics Committee at the Faculty of Medicine of Assiut University (reference number IRB 17100775). The informed consent obtained from the participants verbally because the study comprised observation of the routine medical care and a review of patients' medication profiles with no intervention imposed by authors.

\section{PATIENTS AND METHODS}

This was a prospective observational study that was conducted on patients admitted to the Coronary Critical Care Unit (CCU) of Assiut University Heart Hospital after undergoing PPCI in the Catheterization Unit (CU) in the same hospital. Each patient was followed for three months between May 2018 and September 2019. The CCU comprised three rooms each has a capacity of 4-6 beds.

Patients were eligible for the study if they were adult and admitted to the CCU with new STEMI and treated with PPCI within 12 hrs after symptoms onset. Patients were diagnosed and treated according to the usual clinical practice in the institution. An electrocardiogram was initially recorded for each patient. After the STEMI diagnosis is confirmed, all patients received PPCI. The PPCI (defined as PCI within the first $12 \mathrm{hrs}$ after onset of symptom) was performed by three physicians via femoral access and manual compression was used after sheath removal.

Patients who had any contraindications to the use of ticagrelor (such as hypersensitivity, active pathological bleeding, history of cerebral hemorrhage, intracranial hemorrhage, hemorrhagic stroke and moderate to severe liver disease) as well as those prescribed fibrinolytic therapy within $24 \mathrm{hrs}$ before PCI or oral anticoagulation therapy or strong CYP3A4 inhibitors such as (e.g. ketoconazole, clarithromycin, nefazodone, ritonavir and atazanavir) therapy were excluded from the study ${ }^{13}$.

The patients' medication profiles were clinically reviewed during the CCU stay and at the discharge by the researcher (first author) who is a trained clinical pharmacist with five years of work expertise at the Cardiology Department at the time of this study.

Patients were then followed for three months after the index date (in-hospital initiation of ticagrelor) using phone interviews. The minimum follow-up period was one month.

During the index admission, a $300 \mathrm{mg}$ loading dose of aspirin was given to all patients who were treatment naïve. All patients were pre-treated with a loading dose of ticagrelor $180 \mathrm{mg}$ before PCI. At the discretion of the attending physician, patients were discharged 
on ticagrelor $90 \mathrm{mg}$ bid or clopidogrel $75 \mathrm{mg}$ daily in addition to aspirin (75-100 $\mathrm{mg}$ once daily).

Therefore, patients who were discharged on clopidogrel and/or stopped ticagrelor during hospitalization - because of severe adverse effects of ticagrelor or any other reason described in the results section- were excluded. Patients who discontinued ticagrelor before one month or lost in follow up within the first month were also excluded (Fig. 1).

All patients administered the following parenteral medications before and during PPCI: iopromide dye, lidocaine, heparin, chlorpheniramine, hydrocortisone and prophylactic antimicrobials (amoxicillin clavulinate and gentamicin). Intracoronary tirofiban antiplatelet was used if needed.

The major prescribed medication classes after PPCI during CCU admission were angiotensin- converting enzyme inhibitors, beta-blockers, statins, nitrates, diuretics, trimetazidine, nicorandil, antacids and antimicrobials. Tirofiban, calcium channel antagonists and ivabradine were prescribed for some patients.

The collected data during hospital stay included demographic details, admission and discharge dates, chief complaint, diagnosis, patient and family history of cardiovascular diseases, comorbid conditions, hemodynamic measurements and other laboratory investigations, encountered ticagrelor-related adverse effects, and causes of death (if any).

Patients without complications are often discharged within 2-3 days after admission if they had fulfilled the discharge criteria.

\section{Study outcomes}

The primary efficacy outcome for ticagrelor treatment was evaluated by reporting the incidence of major adverse cardiovascular events (MACE), which is a composite endpoint of death from vascular causes, MI, or ischemic stroke at three months follow-up. The primary safety outcome for ticagrelor was the incidence of major bleeding. Secondary efficacy outcome was the occurrence of stent thrombosis and MI alone. Secondary safety outcome was severe dyspnea, dyspnea led to ticagrelor discontinuation and minor bleeding. Ticagrelor-associated dyspnea was identified using the criteria published by Parodi et al.
$2015^{14}$. Medical events recorded in hospital were not included in primary and secondary outcome measures.

\section{Outcome measures}

Because of the observational nature of the study, all the outcome measures including cardiovascular death, myocardial infarction, stent thrombosis and ischemic stroke were diagnosed and documented in patient's profiles by the treating physicians and acknowledged in the study. However, bleeding as a safety outcome is defined according to the Bleeding Academic Research Consortium published in $2011^{15}$. Major fatal bleeding was defined as bleeding that directly causes death with no other explainable cause. Major non-fatal was defined as overt bleeding plus hemoglobin drop $\geq 3$ to $<5 \mathrm{~g} / \mathrm{dL}$ or any transfusion with overt bleeding (type $3 \mathrm{a}$ ) or overt bleeding plus hemoglobin drop $\geq 5 \mathrm{~g} / \mathrm{dL}$ or cardiac tamponade or bleeding requiring surgical intervention for control (excluding dental/nasal/skin/ hemorrhoid) or bleeding requiring intravenous vasoactive drugs (type $3 \mathrm{~b}$ ) or intracranial hemorrhage or intraocular bleed compromising vision (type 3c). Minor bleeding included any bleed requiring medical intervention to stop or treat.

The Thrombolysis in Myocardial Infarction (TIMI) risk score for STEMI was used for mortality risk estimates in the study patients ${ }^{16}$.

\section{RESULTS AND DISCUSSION}

\section{Results}

Of 169 eligible patients for the study, 17 patients were dropped out during the hospital stay. Then further 32 patients left the study before the end of the first month of follow-up. The reasons of taking out those patients are listed in figure 1. A total of 120 patients completed at least one-month study duration, of whom 109 patients completed the three months follow-up. Patients who had prematurely discontinued ticagrelor intake were 11/120 $(9.2 \%)$. The mean follow-up period was 84 days. The age of patients ranged from 30 to 87 years $($ mean $=55.4 \pm 2.06)$ and $76.7 \%$ were males. Illiterate patients and smokers accounted for $52.5 \%$ and $64.2 \%$ of the study population, respectively. Patients diagnosed with anterior STEMI were 59.2\% (Table 1). 


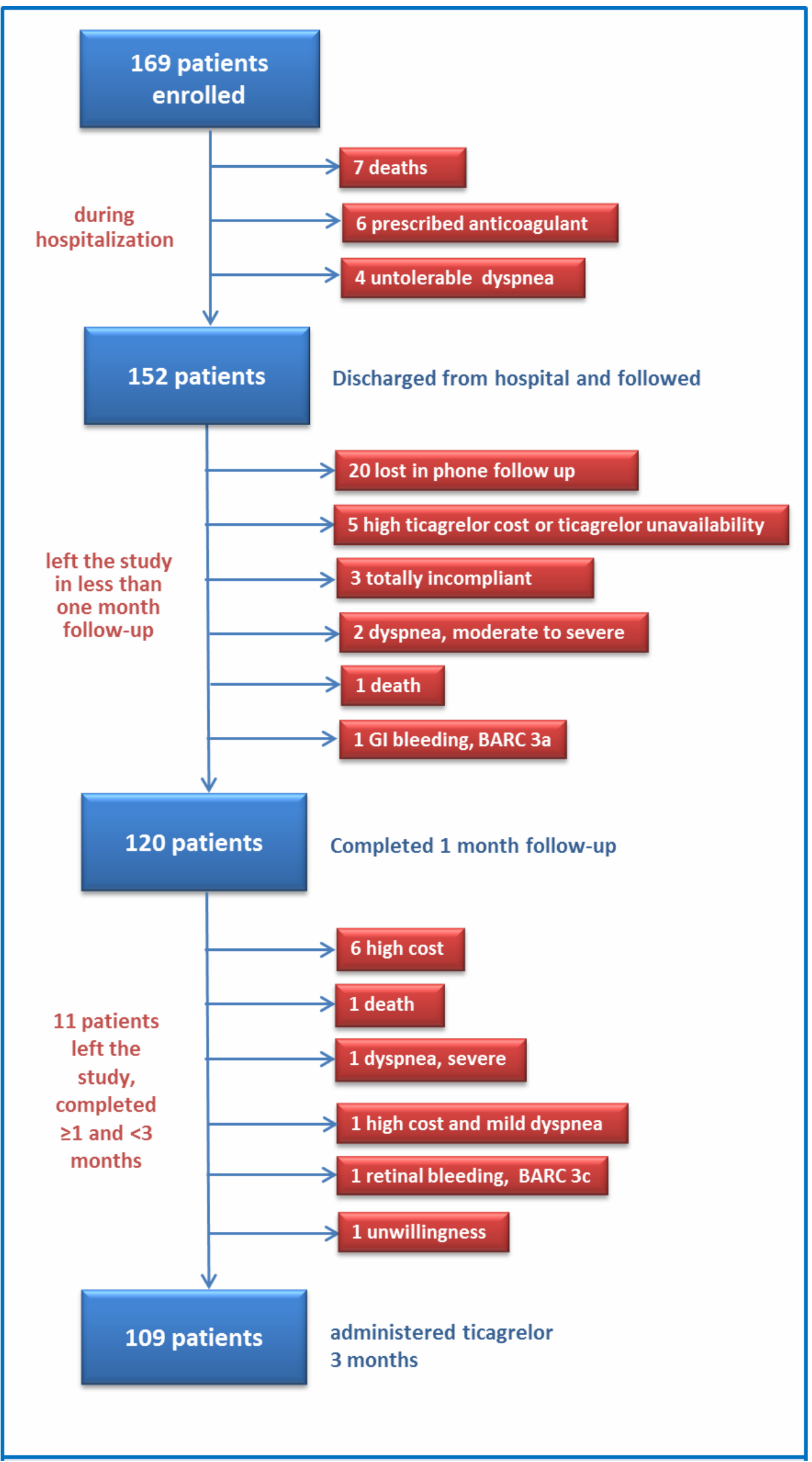

Fig. 1: Flow chart. BARC; Bleeding Academic Research Consortium, GI; Gastrointestinal. 
Table 1: Patients' baseline characteristics.

\begin{tabular}{|c|c|c|}
\hline & $\begin{array}{c}\text { Frequency }(\%) \\
n=120\end{array}$ & $\begin{array}{c}\text { Mean (Standard deviation) } \\
n=120\end{array}$ \\
\hline Age & & $55.4 \pm 2.06$ \\
\hline Weight (kg) & & $79.6 \pm 3.0$ \\
\hline BMI $\left(\mathrm{kg} / \mathrm{cm}^{2}\right)$ & & $28.4 \pm 0.9$ \\
\hline \multicolumn{3}{|l|}{ Sex } \\
\hline Male & $92(76.7)$ & \\
\hline Female & $28(28.3)$ & \\
\hline \multicolumn{3}{|l|}{ Education } \\
\hline Illiterate & $63(52.5)$ & \\
\hline Primary & $4(3.3)$ & \\
\hline Vocation & $14(11.7)$ & \\
\hline University & $26(22.7)$ & \\
\hline \multicolumn{3}{|l|}{ Past medical history } \\
\hline Diabetes Mellitus & $38(31.7)$ & \\
\hline Hypertension & $37(30.8)$ & \\
\hline Cardiovascular & $23(19.2)$ & \\
\hline Previous PCI & $10(8.3)$ & \\
\hline Unstable angina & $8(6.7)$ & \\
\hline Previous MI & $5(4.2)$ & \\
\hline CSA & $5(4.2)$ & \\
\hline Arrhythmia & $1(0.8)$ & \\
\hline Dyspnea & $10(8.3)$ & \\
\hline Liver dysfunction & $3(2.5)$ & \\
\hline Non-hemorrhagic stroke & $3(2.5)$ & \\
\hline \multicolumn{3}{|l|}{ Smoking status } \\
\hline Current smoker & $77(64.2)$ & \\
\hline Heavy smokers & $66(55)$ & \\
\hline Moderate smokers & $10(8.3)$ & \\
\hline Mild smokers & $1(0.8)$ & \\
\hline X-smoker & $7(5.8)$ & \\
\hline \multicolumn{3}{|l|}{ STEMI type } \\
\hline Anterior & $71(59.2)$ & \\
\hline Inferior & $30(25)$ & \\
\hline Infero-posterior & $19(15.8)$ & \\
\hline \multicolumn{3}{|l|}{ Culprit vessel } \\
\hline LAD & $73(60.8)$ & \\
\hline RCA & $33(27.5)$ & \\
\hline LCX & $13(10.8)$ & \\
\hline
\end{tabular}

BMI: body mass index, CSA: central sleep apnea, MI: myocardial infarction, LCX: left circumflex artery, LAD: left anterior descending artery, PCI: percutaneous coronary intervention, RCA: right coronary artery, STEMI: ST-segment elevation myocardial infarction. 


\section{Comorbidities and disease prognosis of the followed sample}

The majority of patients suffered from multiple concurrent comorbidities. The most common were diabetes $(31.7 \%)$, hypertension $(30.8 \%)$ followed by cardiovascular diseases $(19.2 \%)$ and other comorbidities as shown in table 1 .
The TIMI risk score for the enrolled STEMI patients was $>2$ points in $31.7 \%$ of patients. Only two patients were killip-classed more than 2. Risk scores, laboratory data and medical events/procedures during hospitalization are shown in table 2.

Table 2: Risk scores, laboratory data and medical events/procedure during hospitalization.

\begin{tabular}{|c|c|c|}
\hline & $\begin{array}{l}\text { Frequency }(\%) \\
\quad(n=124)\end{array}$ & $\begin{array}{c}\text { Mean (Standard deviation) } \\
(n=124)\end{array}$ \\
\hline \multicolumn{3}{|l|}{ Killip score } \\
\hline Killip I & $107(89.2)$ & \\
\hline Killlip II & $7(5.8)$ & \\
\hline Killip III & $1(0.8)$ & \\
\hline Killip IV & $1(0.8)$ & \\
\hline Killip class $>2$ & $2(1.7)$ & \\
\hline \multicolumn{3}{|l|}{ TIMI risk score } \\
\hline TIMI 0-1 & 30 & \\
\hline TIMI 2 & 48 & \\
\hline TIMI 3 & 17 & \\
\hline TIMI 4 & 14 & \\
\hline TIMI 5 & 4 & \\
\hline TIMI 6-7 & 3 & \\
\hline TIMI STEMI risk score >2 & $38(31.7)$ & \\
\hline Serum Creatinine $(\mu \mathrm{mol} / \mathrm{L})$ & & $99 \pm 7.3$ \\
\hline White blood cells $\left(\mathrm{X} 10^{3} / \mu \mathrm{L}\right)$ & & $10.7 \pm 0.8$ \\
\hline Hemoglobin $(\mathrm{mg} / \mathrm{dL})$ & & $13.4 \pm 0.3$ \\
\hline Platelet count $\left(10^{3} / \mathrm{L}\right)$ & & $242.0 \pm 13.4$ \\
\hline Prothrombin Time & & $17.7 \pm 3.31$ \\
\hline Prothrombin concentration & & $80.6 \pm 3.6$ \\
\hline INR & & $1.1 \pm 0.0$ \\
\hline Medical events/procedure & $26(21.7)$ & \\
\hline Total bleeding & $11(9.2)$ & \\
\hline Minimal (BARC type 1) & $7(5.8)$ & \\
\hline Minor (BARC type 2) & $3(2.5)$ & \\
\hline Major non-fatal (BARC type 3) & $1(0.8)$ & \\
\hline Dyspnea & $10(8.3)$ & \\
\hline Bradycardia & $8(6.7)$ & \\
\hline Heart block & $8(6.7)$ & \\
\hline Second look & $6(5)$ & \\
\hline Stent thrombosis & $4(3.3)$ & \\
\hline Coronary angiography & $3(2.5)$ & \\
\hline Pacemaker insertion & $1(0.8)$ & \\
\hline
\end{tabular}

BARC: bleeding academic research consortium, INR: Internal Normalized Ratio, STEMI: ST-Elevation Myocardial Infarction, TIMI: Thrombolysis in Myocardial Infarction. 


\section{Patients excluded during hospitalization}

Seven patients died during the first five days of hospital stay after receiving ticagrelor. One death was during revascularization as a result of ventricular fibrillation and cardiac arrest. Others died after revascularization. The cause of death was as follows: Two (2/7) patients had cardiac arrest as a consequence of bradycardia; 2/7 patients had cardiogenic shock; 1/7 patient had respiratory failure and one patient's death details were unavailable.

Further, six patients were excluded because of prescribing anticoagulation during hospital stay; of whom 3/6 patients developed atrial fibrillation, 2/6 patients had dilated right atrium $\geq 5.5 \mathrm{~cm}$ and $1 / 6$ patient's echocardiography revealed LV thrombus.

Four patients complained moderate to severe dyspnea and were diagnosed as ticagrelor-related dyspnea so switched to clopidogrel. All the above mentioned 17 patients were excluded from the study.

\section{Exclusion before one month of the index event}

Further 32/152 patients left the study during the first month as follows: 20/152 patients were lost in phone follow-up. Five (5/152) patients had stopped drug administration by physician decision and were switched to clopidogrel due to the high cost of ticagrelor or due to drug unavailability in rural pharmacies. Three (3/152) patients were found incompliant to ticagrelor intake.

\section{Efficacy outcomes at 3 months follow-up}

A total of 120 patients included in the study; 109 patients completed three months follow-up. The primary endpoint occurred in $11 / 120 \quad(9.2 \%)$ of patients receiving aspirin/ticagrelor DAPT therapy, which was a composite of death from CVD 4.2\% (5/120), MI $4.2 \%(5 / 120)$ and ischemic stroke $0.8 \%$ $(1 / 120)$.

Secondary endpoints, such as stent thrombosis occurred in $1.7 \%(2 / 120)$ of patients. No observed cases of transient ischemic attacks (TIAs) or thrombotic events.

\section{Safety outcomes at three months follow-up}

There was nil fatal bleeding and CABGrelated bleeding. Two cases $(1.7 \%)$ suffered from major non-fatal bleeding. One had vaginal bleeding (BARC type 3a) who received one pack RBC transfusion and the other had GI ulcer bleeding (BARC type 3a) which necessitated transfusion of two RBCs packs. Three cases $(2.4 \%)$ of minor bleeding were observed as follows: procedural site bleeding, subcutaneous bleeding (hematoma) and GI bleeding.

Total bleeding was observed in 25/120 (20.8\%) of the followed patients from 12 bleeding sites which were presented as epistaxis, subcutaneous or dermal, hemoptysis, procedural site, oral, post-procedural site, retinal, GI ulcer, hematemesis, hemorrhoidal, urinary tract and vaginal bleeding respectively. The majority of bleedings were minimal (BARC type 1) mostly presented as epistaxis (Table 3).

Multivariate regression analysis of bleeding risk displayed no association with the patients' sex $(p=0.51)$; patients' age of 65 years or older $(p=0.77)$, nor with glycoprotein IIb/IIIa inhibitor GPI (tirofiban) administration during hospitalization $(p=0.77)$.

Dyspnea was the chief complaint in the study and varied in severity. Most reported dyspnea cases were mild. Six cases (6/52) complained of severe dyspnea, of whom 3/6 patients stopped ticagrelor; one stopped medication without replacement and two patients were switched to clopidogrel. The other 3/6 cases suffered ticagrelor-related severe dyspnea but continued medication; one of them caused the patient to rush to the ER twice and despite three hospital clinic visits, physicians didn't switch treatment to another DAPT.

\section{Switching from ticagrelor to other P2Y12 inhibitors}

Figure 1 illustrates that since the start of patients' enrollment, 31 out of 169 (18.3\%) patients were switched from ticagrelor to the P2Y12 inhibitors clopidogrel. The causes of $18.3 \%$ ticagrelor-to-clopidogrel de-escalation were reported as $38.7 \%$ (12/31) high ticagrelor cost, $22.6 \%$ (7/31) dyspnea, $19.3 \%$ (6/31) anticoagulant prescription (exclusion criterion), $12.9 \%$ (4/31) drug noncompliance and $6.5 \%$ (2/31) major non-fatal bleeding. 
Table 3: Clinical efficacy and safety outcomes at three months; reported undesirable effects.

\begin{tabular}{||l|c||}
\hline & $\begin{array}{c}\text { Frequency }(\%) \\
(n=120)\end{array}$ \\
\hline Cardiovascular mortality & $5(4.2)$ \\
\hline Cardiac death and MI & $4(3.3)$ \\
\hline Unknown cause & $1(0.8)$ \\
\hline Cardiac events & $12(10)$ \\
\hline UA & $4(3.3)$ \\
\hline STEMI & $3(2.5)$ \\
\hline NSTEMI & $2(1.7)$ \\
\hline Post MI angina & $2(1.7)$ \\
\hline Stent thrombosis & $2(1.7)$ \\
\hline In-stent restenosis & $2(1.7)$ \\
\hline Ischemic stroke & $1(0.8)$ \\
\hline CABG & $1(0.8)$ \\
\hline Bleeding severity & \\
\hline Minimal & $25(20.8)$ \\
\hline Minor & $3(2.5)$ \\
\hline Major, non-fatal & $2(1.7)$ \\
\hline Dyspnea, all & $52(43.3)$ \\
\hline Mild & $31(25.8)$ \\
\hline Moderate & $15(12.5)$ \\
\hline Severe & $6(5)$ \\
\hline Led to discontinuation & $2(1.7)$ \\
\hline Undesirable adverse effects & $82(68.3)$ \\
\hline Dyspnea & $52(43.3)$ \\
\hline Fatigue & $32(26.7)$ \\
\hline Total bleedings & $29(24.2)$ \\
\hline Headache & $20(16.7)$ \\
\hline Dizziness & $13(10.8)$ \\
\hline Hypertension & $6(5)$ \\
\hline Arthralgia & $4(3.3)$ \\
\hline Cough & $4(3.3)$ \\
\hline Gastritis & $4(3.3)$ \\
\hline Hypotension & $4(3.3)$ \\
\hline Dyspepsia & $3(2.5)$ \\
\hline Back pain & $2(1.7)$ \\
\hline Insomnia & $2(1.7)$ \\
\hline Parasthesia & $2(1.7)$ \\
\hline Vertigo & \\
\hline Vomiting & \\
\hline Others & \\
\hline \hline & \\
\hline
\end{tabular}

Note: Undesirable effects reported maybe contributed to medications other ticagrelor.

\section{Discussion}

This observational study explored the efficacy and safety of ticagrelor in a group of patients indicated for urgent revascularization.

The findings recorded a primary endpoint - a composite of death from vascular causes, MI, or ischemic stroke, at 3 months in $9.2 \%$ of patients. The calculated net clinical benefit which was the composite of cardiovascular death, unplanned hospitalization leading to urgent coronary revascularization, stroke, and bleeding episodes (defined by the BARC classification $\geq 2$ ) occurred in $10.7 \%$ of patients. Bleeding events in this study excluding minimal bleeding were reported in $4.2 \%$ of patients. Ticagrelor was also associated with a low incidence of major bleeding and a high incidence of dyspnea which was the main cause of drug discontinuation.

The primary endpoint was obviously higher than that of ticagrelor arm of the STEMI intended for PPCI subgroup analysis of the PLATO study at 3 months $(6.2 \%)^{11}$; however, it was close to the cumulative MACE incidence at 12 months $(9.4 \%)^{11}$ and that reported in the PHILO study (9\%) which enrolled Asian patients ${ }^{17}$. The incidence was also higher than other reported in some studies; The Efficacy and Safety of Ticagrelor versus clopidogrel in Acute coronary syndrome observational pilot study in Taiwan (ESTATE) ${ }^{18}$, in which primary efficacy outcome was $7.1 \%$. The Greek AntiPlatElet registry (GRAPE) ${ }^{19}$ reported a primary endpoint of $6.8 \%$. In the CHANGE DAPT study $^{20}$, the primary endpoint was onehalf lower $(4.7 \%)$ compared to this study. The higher primary outcome in this study may be related to the high number of patients left the study or switched to clopidogrel before the end of three months, thus it may not reflect the true efficacy of ticagrelor.

The calculated net clinical benefit occurred in $10.7 \%$ of patients was much lower than net clinical benefit $(26.3 \%)$ of the (TOPIC) study $^{21}$ and may be explained by the less observed BARC grade $\geq 2$ cases in this study.

Cardiovascular mortality incidence $(4.2 \%)$ in this study was higher as compared to the STEMI PLATO study $(3 \%)^{11}$, the GRAPE registry $(3.1 \%)^{19}$, the PHILO study $(2.2 \%)^{17}$, the TOPIC $(1.2 \%)^{21}$ and CHANGE DAPT studies $(2.9 \%)^{20}$; however, it was close to the 
vascular mortality rate $(4.5 \%)$ reported in the ESTATE study ${ }^{18}$. This may be attributed to the small sample size of this study and different statistical power.

The observed myocardial infarction rate $(4.2 \%)$ in this study was more than that reported in previous studies such as the STEMI patients intended for PPCI subgroup analysis of the PLATO study $(3 \%)^{11}$, the ESTATE $(3.1 \%)^{18}$, the CHANGE DAPT $(2.2 \%)^{20}$ and the GRAPE $(1.1 \%)^{19}$ studies. However, the rate was less than reported in the PHILO study $(6 \%)^{17}$ and the unchanged DAPT arm in the TOPIC study $(9.3 \%)^{21}$.

The incidence of ischemic stroke $(0.8 \%)$ were comparable to that of the PLATO ${ }^{11}$, ESTATE $^{18}$, GRAPE registry ${ }^{19}$, CHANGE DAPT $^{20}$ and TOPIC studies ${ }^{21}(1 \%, 0.4 \%, 0.7 \%$, and $0.9 \%$ and $1.1 \%$ respectively). However, the stroke incidence was less than that reported in the PHILO study $(2.2 \%)^{17}$.

Stent thrombosis rate was lower than the STEMI PLATO subgroup analysis ${ }^{11}$; 3.3\%, while the rate was higher as compared to the CHANGE DAPT $(0.8 \%)^{20}$.

Bleeding events in this study $(4.2 \%)$ excluding minimal bleeding, which is bleeding not prompt medical attention, were three times lower than reported in the STEMI arm of PLATO subgroup analysis study $(13.1 \% \text {. })^{11}$. We observed no major fatal bleeding (BARC type 5) compared to $4.7 \%$ of PLATO subgroup analysis and no major CABG-related bleeding (BARC type 4) compared to 5.1\%. Major nonfatal bleeding incidence (BARC type 3) was about five times lower compared to $8.2 \%$. Minor bleeding (BARC type 2) was lower to half compared to $4.9 \%$ of PLATO study. The majority of bleeding events in this study were minimal (BARC type 1). Minimal bleeding was not reported in the PLATO study ${ }^{11}$ and $7.3 \%$ required blood transfusion versus two cases $(1.7 \%)$ in this study.

No fatal bleeding (BARC type 5) was observed compared to $0.4 \%$ in ESTATE population and minor bleeding (BARC type 2) was $2.5 \%$ compared to $6.3 \%$ in ESTATE population ${ }^{18}$. The total bleeding and minimal bleeding rates in our study population were higher in comparison with the Eastern Asians, however, with less bleeding sites (12 versus 44 bleeding sites in ESTATE population) ${ }^{18}$.
However, the total bleeding was much lower in this study compared to the GRAPE study $^{19}$; total BARC and BARC type 1 were one-half lower than the GRAPE study (BARC 2 was less by $2.3 \%$ and BARC $\geq 2$ was 2.6 times less).

The incidence of major and minor bleeding in this study was four times lower and six times lower than that of the PHILO study's major and minor non-CABG related bleeding, $(8.3 \% \text { and } 15.2 \% \text {, respectively })^{17}$. This finding may give clinicians some convenience to use ticagrelor without the big concern about major bleeding and its associated morbidity and mortality in Egyptian patients

Quite the contrary, the incidence dyspnea was more than seven-fold higher than that reported in the PHILO study ${ }^{17}$; more than three-fold higher than reported in the STEMI PLATO population ${ }^{11}$ and was double that of the findings of the ESTATE study ${ }^{18}$.

The incidence of dyspnea of any grade of severity -observed at three months- was consistent with that of the Australian study of the real-world incidence of patient-reported dyspnea with ticagrelor ${ }^{22}$. However, this study reported lower proportions of drug discontinuations; $1.7 \%$ versus $7.14 \%$. This seems because of many physicians in this study to continued ticagrelor therapy despite dyspnea symptoms because some patients encountered comorbidities and were at moderate to high risk of re-infarction.

Considering a future study of ticagrelor de-escalation after the early phase of ischemic event may be beneficial in reducing the incidence of dyspnea and reducing prescription cost, thus minimizing drug malcompliance.

\section{Limitations of the study}

The findings of this study need to be viewed in light of some limitations. The principal limitation was the nature of study design as an observational study of physician practice without researcher intervention and therefore, the lack of a comparison group clopidogrel group - and randomization. Assiut hospital revascularization management protocol stipulates the use of ticagrelor for its faster than clopidogrel- onset of action, which in turn affects the results' strength. Second is the short duration of patient follow-up and small sample size, which may affect the drawn 
conclusion and results' significance on ticagrelor efficacy. This was because of many patients' attrition after one month and before the three months follow-up as they were switched to clopidogrel or left the study. Eventually, many results at three months were compared to 12 months-results of previous studies due to their lack of detailed percentages at different follow-up periods.

\section{Statistical analysis}

Patients' baseline characteristics were summarized as mean, standard deviations and 95\% CI for continuous variables such as age, weight, body mass index and laboratory measurements. Categorical variables such as sex, education, and comorbidities were summarized as proportions. Multivariate regression analysis was performed for the association between safety outcomes (bleeding and dyspnea) and demographic and medicinerelated factors.

All the statistical analyses were performed using SPSS version 22 (SPSS Inc., Chicago, IL, USA). The significance level was set at $p$ value $<0.05$.

\section{Conclusions}

Ticagrelor induced a composite of death from vascular causes, MI, and ischemic stroke that was higher than those reported in some previous studies. Ticagrelor was also associated with a much lower incidence of major bleeding despite using femoral access approach in PPCI and a higher incidence of dyspnea than previously reported. Many of dyspnea cases necessitated drug discontinuation than bleeding did with no effect on the mortality rate.

\section{REFERENCES}

1- World Health Organization, "The top 10 causes of death", [Cited 15 February 2019], Available from: https://www.who. int/news-room/fact-sheets/detail/the-top10-causes-of-death (2018).

2- A. N. Nowbar, J. P. Howard, J. A. Finegold, P. Asaria and D. P. Francis, "Global geographic analysis of mortality from ischemic heart disease by country, age and income: Statistics from World Health Organisation and United Nations",
International Journal of Cardiology, 174 (2), 293-8 (2014).

3- M. Valgimigli, H. Bueno, R. A. Byrne, JP. Collet, F. Costa, A. Jeppsson, et al., "ESC focused update on dual antiplatelet therapy in coronary artery disease developed in collaboration with EACTS: The Task Force for dual antiplatelet therapy in coronary artery disease of the European Society of Cardiology (ESC) and of the European Association for Cardio-Thoracic Surgery (EACTS)", European Heart Journal, 39 (3), 213-260 (2017).

4- S. Fathy, M. H. Shahin, T. Langaee, B. M. Khalil, A. Saleh, N. A. Sabry, et al., "Pharmacogenetic and clinical predictors of response to clopidogrel plus aspirin after acute coronary syndrome in Egyptians", Pharmacogenetics and Genomics, 28 (9), 207-213 (2018).

5- M. Pavlovic, S. Apostolovic, D. Stokanovic, J. Lilic, S. S. Konstantinovic, J. B. Zvezdanovic, et al., "The association of clopidogrel and 2-oxo-clopidogrel plasma levels and the 40 months clinical outcome after primary PCI", International Journal of Clinical Pharmacy, 40 (6), 1482-1489 (2018).

6- N. A. Farid, A. Kurihara and S. A. Wrighton, "Metabolism and disposition of the thienopyridine antiplatelet drugs ticlopidine, clopidogrel, and prasugrel in humans", J. Clin. Pharmacol., 50 (2), 126-42 (2010).

7- B. M. Khalil, M. H. Shahin, M. H. M. Solayman, T. Langaee, M. F. Schaalan, Y. Gong, et al., "Genetic and Nongenetic Factors Affecting Clopidogrel Response in the Egyptian Population", Clinical and Translational Science, 9 (1), 23-28 (2016).

8- V. L. Serebruany, S. R. Steinhubl, P. B. Berger, A. I. Malinin, D. L. Bhatt and E. J. Topol, "Variability in platelet responsiveness to clopidogrel among 544 individuals", Journal of the American College of Cardiology, 45 (2), 246-251 (2005).

9- G. N. Levine, E. R. Bates, J. C. Blankenship, S. R. Bailey, J. A. Bittl, B. Cercek, et al., "ACCF/AHA/SCAI guideline for percutaneous coronary 
intervention", Circulation, 124 (23), e574e651 (2011).

10- S. Husted and J. J. J. Van Giezen, "Ticagrelor: The first reversibly binding oral P2Y12 receptor antagonist", Cardiovascular Therapeutics, 27 (4), 259-274 (2009).

11- P. G. Steg, S. James, R. A. Harrington, D. Ardissino, R. C. Becker, C. P. Cannon, et al., "Ticagrelor versus clopidogrel in patients with ST-elevation acute coronary syndromes intended for reperfusion with primary percutaneous coronary intervention. A platelet inhibition and patient outcomes (PLATO) trial subgroup analysis", Circulation, 122 (21), 21312141 (2010).

12- L. Wallentin, R. C. Becker, A. Budaj, C. P. Cannon, H. Emanuelsson, C. Held, et al., "Ticagrelor versus clopidogrel in patients with acute coronary syndromes", New England Journal of Medicine, 361 (11), 1045-1057 (2009).

13- B. Lombo and J. G. Díez, "Ticagrelor: the evidence for its clinical potential as an oral antiplatelet treatment for the reduction of major adverse cardiac events in patients with acute coronary syndromes", Core Evidence, 6, 31-42 (2011).

14- G. Parodi and R. F. Storey, "Dyspnea management in acute coronary syndrome patients treated with ticagrelor", European Heart Journal: Acute Cardiovascular Care, 4 (6), 555-560 (2014).

15- R. Mehran, S. V. Rao, D. L. Bhatt, C. M. Gibson, A. Caixeta, J. Eikelboom, et al., "Standardized bleeding definitions for cardiovascular clinical trials", Consensus Report From the Bleeding Academic Research Consortium, 123 (23), 27362747 (2011).

16- D. A. Morrow, E. M. Antman, A. Charlesworth, R. Cairns, S. A. Murphy, J. A. D. Lemos, et al., "TIMI risk score for ST-elevation myocardial infarction: A convenient, bedside, clinical score for risk assessment at presentation", Circulation, 102 (17), 2031-2037 (2000).
17- S. Goto, C-H. Huang, S-J. Park, H. Emanuelsson and T. Kimura, "Ticagrelor vs. clopidogrel in Japanese, Korean and Taiwanese patients with acute coronary syndrome - randomized, double-blind, Phase III PHILO Study", Circulation Journal, 79 (11), 2452-2460 (2015).

18- I. C. Chen, C. H. Lee, C. C. Fang, T. H. Chao, C. L. Cheng, Y. Chen, et al., "Efficacy and safety of ticagrelor versus clopidogrel in acute coronary syndrome in Taiwan: A multicenter retrospective pilot study", J. Chin. Med. Assoc., 79 (10), 521-30 (2016).

19- D. Alexopoulos, I. Xanthopoulou, S. Deftereos, M. Hamilos, G. Sitafidis, I. Kanakakis, et al., "Contemporary antiplatelet treatment in acute coronary syndrome patients undergoing percutaneous coronary intervention: 1Year outcomes from the Greek AntiPlatelet (GRAPE) Registry", Journal of Thrombosis and Haemostasis, 14 (6), 1146-1154 (2016).

20- C. von Birgelen, P. Zocca, L. van der Heijden, M. Kok, M. Stoel, M. Hartmann, et al., "TCT-549 CHANGE DAPT: Clopidogrel or ticagrelor in acute coronary syndrome patients treated with newergeneration drug-eluting stents", Journal of the American College of Cardiology, 70 (18 Supplement), B227 (2017).

21- T. Cuisset, P. Deharo, J. Quilici, T. Johnson, S. Deffarges, C. Bassez, et al., "Benefit of Switching Dual Antiplatelet Therapy After Acute Coronary Syndrome: The TOPIC (Timing of Platelet Inhibition After Acute Coronary Syndrome) Randomized Study", Vol. 38 (2017).

22- A. E. Prosser, J. L. Dawson, K. Koo, K. M. O'Kane, M. B. Ward, R. J. Woodman, et al., "Real-world incidence of patientreported dyspnea with ticagrelor", Therapeutic Advances in Drug Safety, 9 (10), 577-584 (2018). 


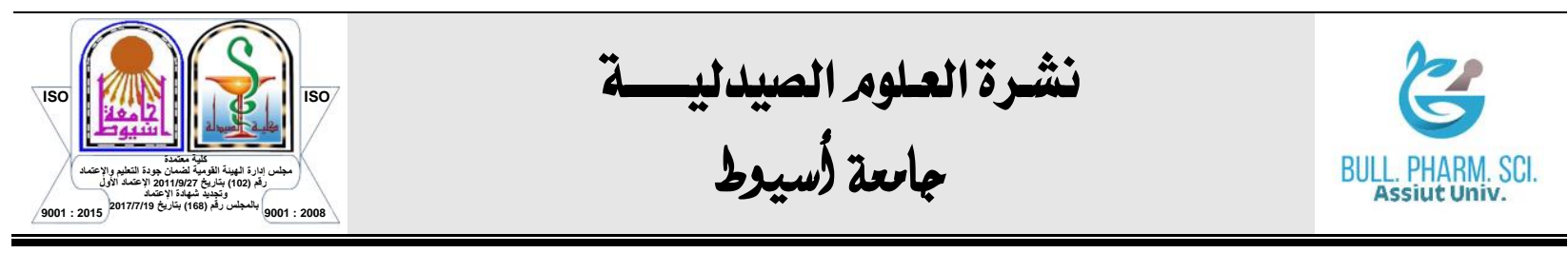

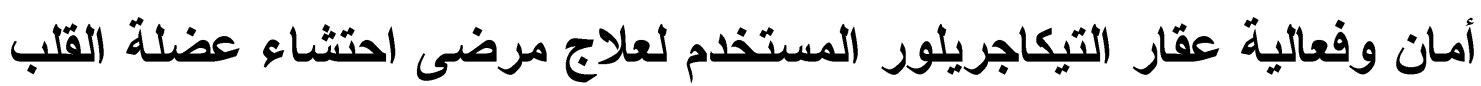

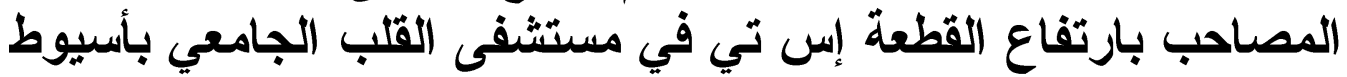

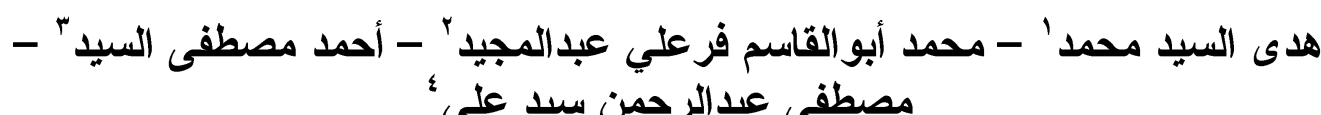
'صيدلي اكلينيكي بعناية القلب المركزة ، مستشفى الأورمان الجامعي للقلب ، أسيوط ، مصر البيل

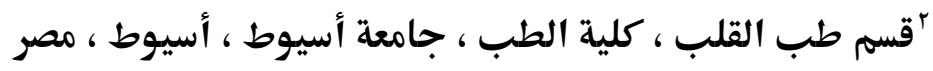

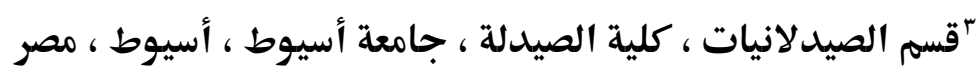

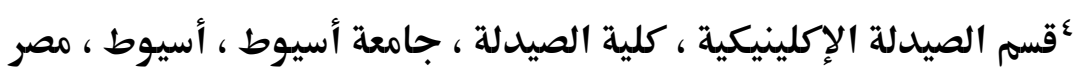

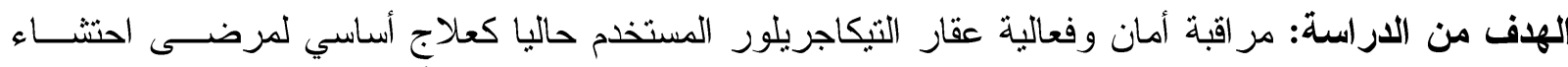
عضلة القلب الحاد المصاحب بارتفاع القطعة إس تي في مستثنفى القلب الجامعي بأسيوط. المكان: وحدة العناية المركزة لمرضى القلب بمستشفى القلب الجامعي بأسيوط.

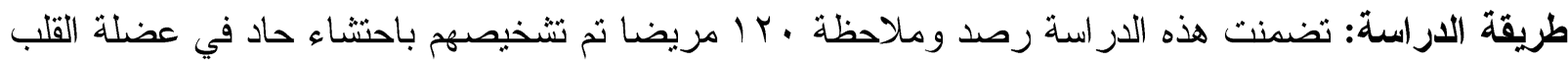

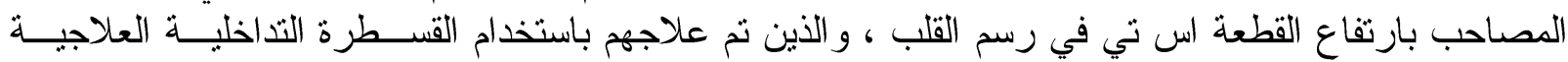

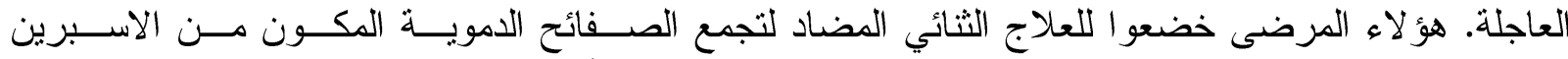

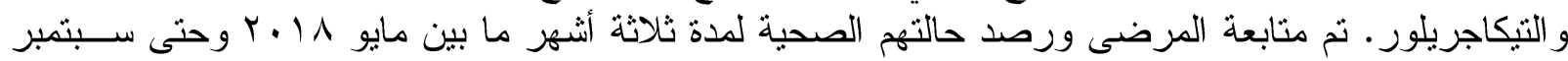

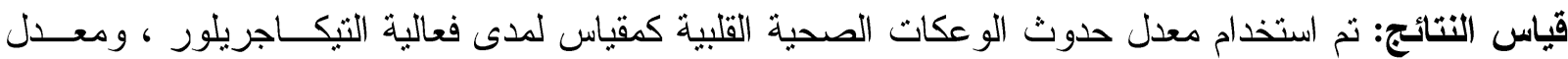

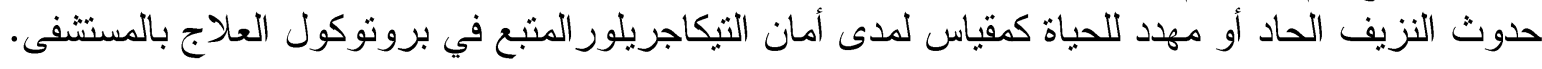

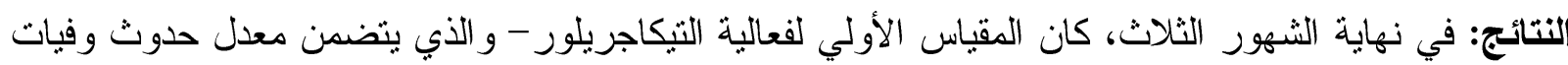

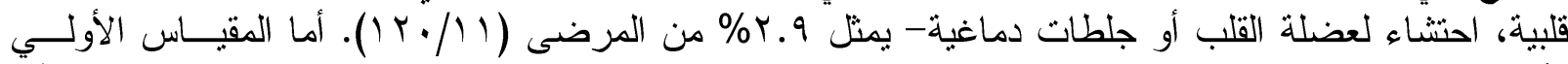

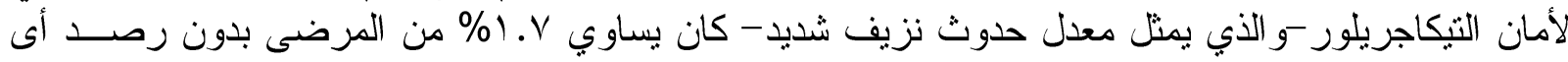

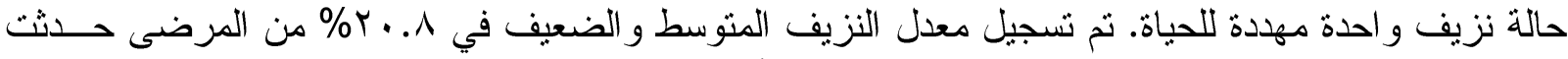

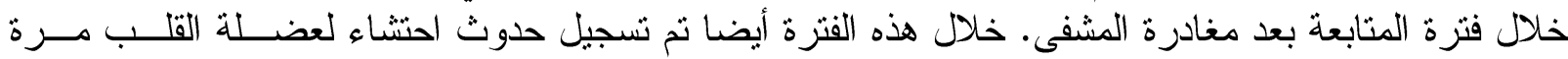

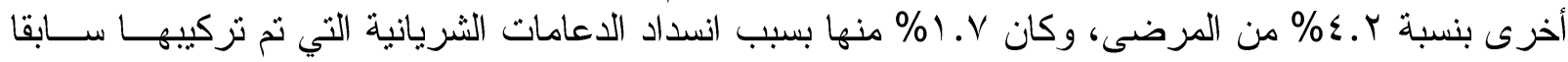

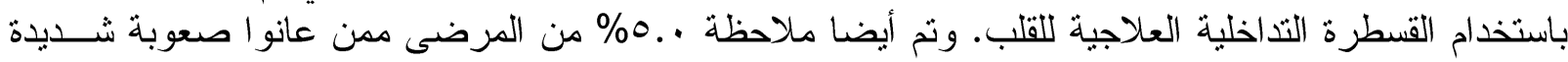

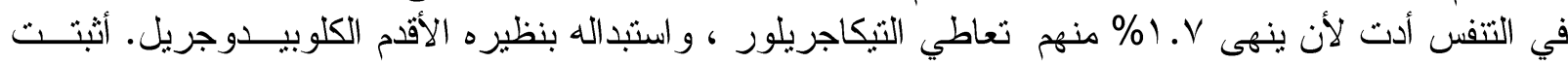

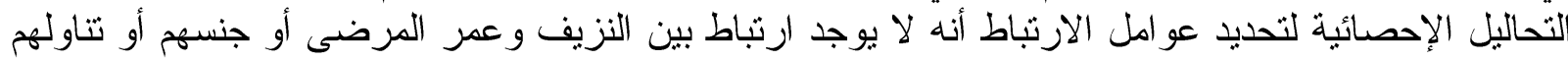

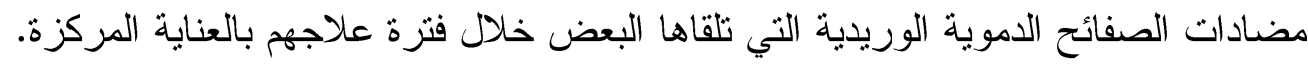

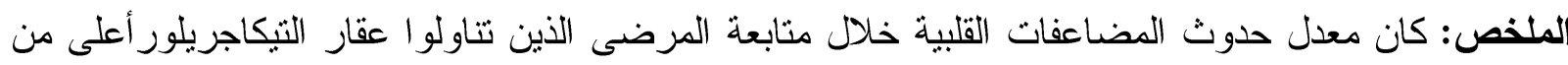

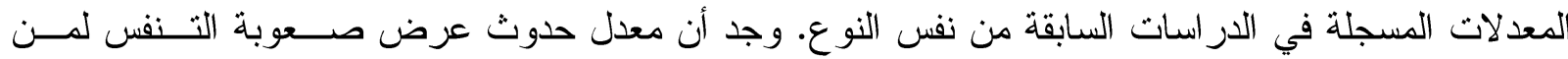

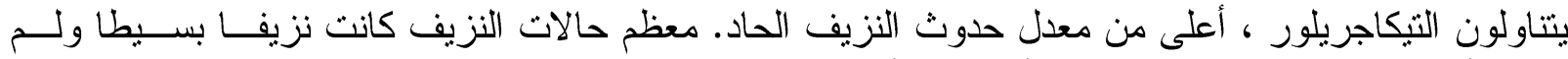

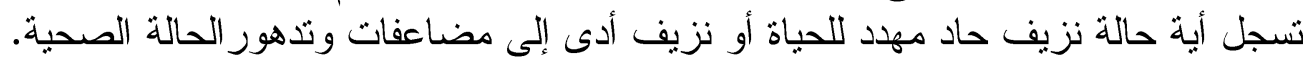

